Respiration 2010;79:85-86

DOI: $10.1159 / 000232395$

\section{Primary Coccidioidal Pleural Effusions Successfully Treated with Adjunctive Corticosteroid Therapy}

René Agustín Flores-Franco, Rocío Aguayo-Yong

Department of Internal Medicine, Hospital General Regional de Zona No. 1, Unidad 'Morelos', Instituto Mexicano del Seguro Social, Chihuahua, México

We read with great interest the case report of Swartz et al. [1] about acute eosinophilic pneumonia due to coccidioidomycosis, which was successfully treated with adjuvant corticosteroids. This and another report [2] have made important contributions to the scarce literature related to corticosteroid use in coccidioidomycosis. Corticosteroids have been successfully used in the treatment of coccidioidomycosis associated with acute respiratory syndrome, but not in the treatment of other forms of complicated coccidioidomycosis. This justification for corticosteroid use has come about in a situation considered invariably lethal and without any therapeutic alternatives [2]. Actually, the role of adjuvant therapy with corticosteroids in the treatment of coccidioidomycosis is not recommended by the current guidelines due to the risks of microbial dissemination [3]. Here, we describe a patient with bilateral pleural effusions attributed to coccidioidomycosis in whom the use of systemic corticosteroids combined with azole therapy brought about a more rapid improvement in symptoms and reabsorption of fluid.
A 69-year-old male patient came to our center from an area well known to be endemic for coccidioidomycosis. He had a 1month history of progressive dyspnea, mild cough, chest pain with occasional night fever and sweats. Chest X-ray and a computed tomography showed bilateral and large pleural effusions without lung opacities (fig. 1a). Initial laboratory results revealed: hemoglobin level $14.3 \mathrm{~g} / \mathrm{dl}$; blood leukocyte count $10^{9} \mathrm{cells} / \mu \mathrm{l}$; polymorphonuclear count 6,700 cells $/ \mu \mathrm{l}$; lymphocytes 1,500 cells/ $\mu$ l, and eosinophils 1,900 cells/ $\mu$ l. An HIV test was negative. Therapeutic thoracocentesis was conducted on the right side and chemistry fluid analysis displayed: glucose $63 \mathrm{mg} / \mathrm{dl}$; protein 6 $\mathrm{g} / \mathrm{dl}$, and lactate dehydrogenase $327 \mathrm{UI} / \mathrm{l}$. The cytological analysis gave a cell count of 2,430 leukocytes, 53\% lymphocytes, and $45 \%$ eosinophils. Bacterial gram stain and culture, acid-fast bacilli smears, fungal culture and cytology were all negative. A pleural closed-biopsy performed during the same procedure gave evidence of fibrosis and edema of parietal pleura and infiltrated by lymphocytes, macrophages, eosinophils, and plasmatic cells. Then, left-sided pleuroscopy was performed which showed diffuse thickening of the parietal pleura and fibrinous adhesions. The pleural biopsy obtained by this means showed inflammatory changes with eosinophils predominating in the cell infiltration, but no granulomas or microorganisms were identified. Because no significant relief of constitutional symptoms was obtained with broad antimicrobial therapy, it was decided to initiate antifungal therapy with itraconazole while awaiting confirmatory serological results for coccidioidomycosis. After 1 week of treatment as an outpatient, his symptoms persisted without radiology improvement (fig. 1b), therefore a course of prednisone therapy was added at dose of $0.7 \mathrm{mg} / \mathrm{kg} /$ day. The patient's symptoms quickly began to improve and after 3 weeks notable reabsorption
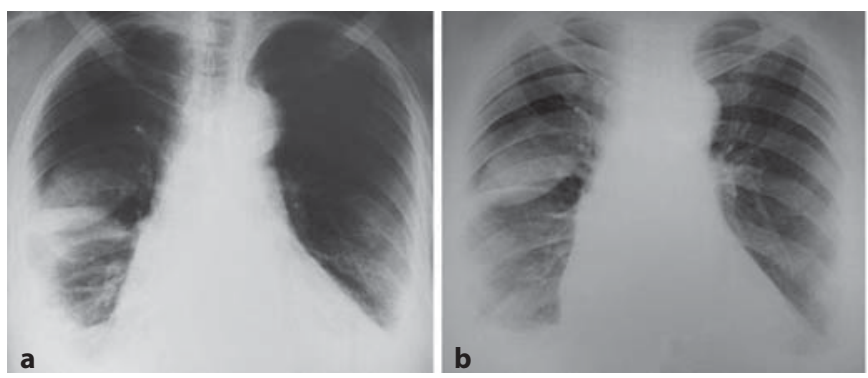

Fig. 1. a Chest radiograph at the beginning of itraconazole therapy and showing bilateral pleural effusions (pleural fluid is seen in the minor fissures). $\mathbf{b}$ After the first week of treatment the chest radiograph remained practically without radiological changes.
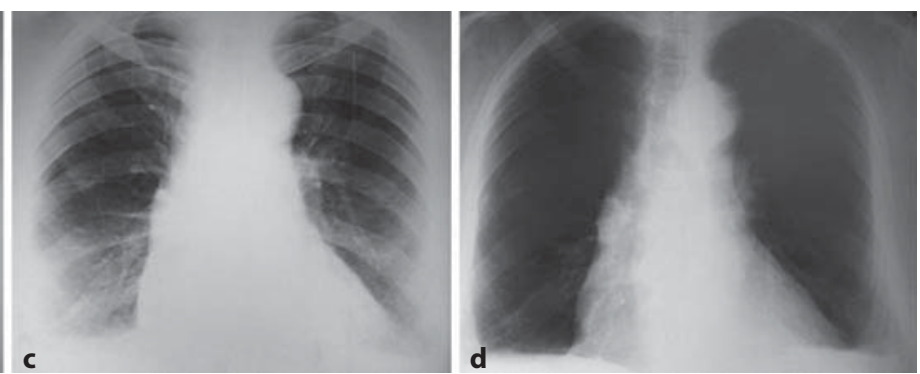

c Chest radiograph taken in the next 3 weeks of corticosteroid therapy showing a significant clearance of pleural fluid. $\mathbf{d}$ Chest radiograph 15 days after the withdrawal of corticosteroid showing resolution of pleural effusions.

\section{KARGER}

Fax +41 613061234 E-Mail karger@karger.ch www.karger.com (c) 2009 S. Karger AG, Base

0025-7931/10/0791-0085\$26.00/0 
of pleural fluid was observed on chest X-ray (fig. 1c). At that time, the requested serologic testing (latex agglutination) was found to be positive for IgM antibodies to Coccidioides immitis antigens. The dose of prednisone was tapered and finally stopped in the next 2 weeks, and after 2 months on itraconazole therapy the patient continued to be asymptomatic and his pleural effusions had resolved (fig. 1d).

In the absence of empyema, pleural effusions associated with coccidioidomycosis are thought to have an acceptable response to oral antifungal therapy alone and usually resolve in few weeks without complications [4]. Nevertheless, there are reports [5-7] on the need for a long course of antifungal therapy and prolonged hospital stay before clinical and radiological improvement occurs. In many instances the physiopathology of coccidioidal pleural effusions resembles that of tuberculosis as fluid collections may result from the direct extension of infection from the lung parenchyma into the pleural space or by means of immune complex pleuritis due to antigens exposure [8]. Theoretically, such a delayed hypersensitivity reaction to Mycobacterium tuberculosis antigens is thought to be the rationale for adjunctive corticosteroid therapy in patients with tuberculosis pleuritis [9]. At the present time, guidelines do not support adjunctive corticosteroid therapy for the treatment of primary pleural coccidioidomycosis probably because it has a better prognosis than tuberculous pleuritis and there is a theoretical risk for dissemination of infection. Our justification for this practice was guided by the clinical findings in a patient with a potential diagnosis of coccidioidomycosis associated with low-risk factors for dissemination, such as the microbiology and histopathology findings which were unable to demonstrate a fungus that would contraindicate corticosteroid treatment.

Considering that delayed hypersensitivity plays a role in determining the occurrence and amount of pleural effusion and the consequent residual pleural thickening, adjuvant therapy with systemic corticosteroids could be useful in shortening the acute phase of the illness and restoring the integrity of the pleura. Nevertheless, although corticosteroids have proven useful in the early relief of acute clinical symptoms in patients with tuberculous pleuritis, controversy still exists about whether corticosteroids are effective in the prevention of residual pleural thickening [9]. Our patient displayed early resolution of pleural effusion which could had prevented the development of residual pleural thickening; however, such a complication is also considered infrequent and pleural thickening has been described only in sporadic patients with coccidioidomycosis [10].
In conclusion, we want to complement the literature by presenting the first case report on the successful response to adjuvant corticosteroid therapy for eosinophilic pleural effusions attributed to $C$. immitis. This case suggests the role of hypersensitivity in the development of reactive pleural effusions in patients with coccidioidomycosis, and thus opens the possibility for further prospective studies before a striking recommendation for the use of adjuvant corticosteroids can be made in the non-immunocompromised patient with a slowed response to conventional antifungal drugs.

\section{References}

1 Swartz J, Stoller JK: Acute eosinophilic pneumonia complicating Coccidioides immitis pneumonia: a case report and literature review. Respiration 2009;77:102-106.

2 Shibli M, Ghassibi J, Hajal R, O’Sullivan M: Adjunctive corticosteroids therapy in acute respiratory syndrome owing to disseminated coccidioidomycosis. Crit Care Med 2002;30:1896-1898.

3 Galgiani JN, Ampel NM, Blair JE, Catanzaro A, Johnson JH, Stevens DA, Williams PL: Coccidioidomycosis. Clin Infect Dis 2005;41:12171223.

4 Sahn SA, Cohen M: Resolution of pleural effusions. Chest 2001;119: 1547-1562.

5 Pinckney L, Parker BR: Primary coccidioidomycosis in children presenting with massive pleural effusion. AJR Am J Roentgenol 1978;130: 247-249.

6 Hamer L, Castillo J: Coccidioidomycosis presenting as a massive pleural effusion in a postpartum woman. Indian J Chest Dis Allied Sci 2006; 48:59-62.

7 Afshar K, Boydking A, Sharma OP: Exudative pleurisy of coccidioidomycosis: a case report and review of the literature. J Med Case Reports 2008;2:291.

8 Crum-Cianflone NF, Truet AA, Teneza-Mora N, et al: Unusual presentations of coccidioidomycosis: a case series and review of the literature. Medicine (Baltimore) 2006;85:263-277.

9 Gopy A, Madhavan SM, Sharma SK, Sahn SA: Diagnosis and treatment of tuberculous pleural effusion. Chest 2006;131:880-889.

10 McGahan JP, Graves DS, Palmer PES, Stadalnik RC, Dublin AB: Classic and contemporary imaging of coccidioidomycosis. AJR Am J Roentgenol 1981;136:393-404.

René Agustín Flores-Franco, MD

Department of Internal Medicine, Hospital General Regional de Zona No. 1, Unidad 'Morelos', Instituto Mexicano del Seguro Social, Universidad y García Conde Av. s/n Col Centro, Chihuahua, Chih., 31000 (México)

Tel. +52 614413 3238, E-Mail rflores99@prontomail.com 